\section{Matrix metalloproteinases in angiogenesis: a moving target for therapeutic intervention}

\author{
William G. Stetler-Stevenson \\ Laboratory of Pathology, Division of Clinical Sciences, National Cancer Institute, \\ Bethesda, Maryland 20892, USA
}

Address correspondence to: William G. Stetler-Stevenson, Extracellular Matrix Pathology Section, Laboratory of Pathology, Division of Clinical Sciences, National Cancer Institute, Building 10, Room 2A33, MSC \#1500, 10 Center Drive, Bethesda, Maryland 20892, USA. Phone: (301) 496-2687; Fax: (301) 402-2628; E-mail: sstevenw@mail.nih.gov.
Angiogenesis is the process in which new vessels emerge from existing endothelial lined vessels. This is distinct from the process of vasculogenesis in that the endothelial cells arise by proliferation from existing vessels rather than differentiating from stem cells. Angiogenesis is an invasive process that requires proteolysis of the extracellular matrix and, proliferation and migration of endothelial cells, as well as synthesis of new matrix components. During embryonic development, both vasculogenesis and angiogenesis contribute to formation of the circulatory system. In the adult, with the single exception of the reproductive cycle in women, angiogenesis is initiated only in response to a pathologic condition, such as inflammation or hypoxia. The angiogenic response is critical for progression of wound healing and rheumatoid arthritis. Angiogenesis is also a prerequisite for tumor growth and metastasis formation. Therefore, understanding the cellular events involved in angiogenesis and the molecular regulation of these events has enormous clinical implications. This understanding is providing novel therapeutic targets for the treatment of a variety of diseases, including cancer.

Whatever the pathologic condition, an initiating stimulus results in the formation of a migrating solid column of endothelial cells called the vascular sprout. The advancing front of this endothelial cell column presumably focuses proteolytic activity to create a defect in the extracellular matrix, through which the advancing and proliferating column of endothelial cells migrates. Behind this advancing front of protease activity, a region of differentiation develops in which the endothelial cells tightly adhere to one another, form a new basement membrane, stop proliferating, and develop a lumen for the new capillary. Fusion of these vascular sprouts establishes blood flow in the newly vascularized region.

The matrix metalloproteinases (MMPs) are a family of extracellular endopeptidases that selectively degrade components of the extracellular matrix. The MMPs are clearly implicated in angiogenesis. The most direct and compelling evidence for this conclusion is that MMP inhibitors, both synthetic and endogenous, inhibit angiogenic responses both in vitro and in vivo (1-4). Moreover, recent studies provide evidence that MMPdeficient mice exhibit delayed or diminished angiogenic responses during development or in response to tumor xenografts $(5,6)$. While these studies clearly implicate functional MMP activity in the angiogenic response, there is some debate regarding the possible molecular targets that are involved and their precise role in angio- genesis. Moreover, the mechanisms that control and integrate MMP activity with other endothelial cell functions are poorly understood. Such functions include endothelial cell attachment to the extracellular matrix, detachment, and migration/invasion. The temporal and spatial relationships of MMP activity to these endothelial cell functions define the angiogenic phenotype.

Understanding what, where, when, and how MMP activity is involved in the angiogenic phenotype has enormous implications for cancer therapy because angiogenesis is necessary for tumor growth and metastasis. Current therapeutic approaches targeting MMP activity utilize general class inhibitors that are selective, but not specific, for some MMP family members. This has resulted in moderately severe, but reversible, musculoskeletal complications. This experience highlights our need for a better understanding of the specific MMPs and their precise role in the angiogenic response. In this way, we can discern what MMPs to target, and when to target them, with the aim of limiting side effects and possible complications.

The MMPs are a family of secreted and membraneassociated neutral endopeptidases with a diverse spectrum of substrates (7). These enzymes are produced by a variety of cell types, including epithelial cells, fibroblasts, and inflammatory cells. MMPs reportedly produced by endothelial cells are MMP-1, MMP-2, MMP-9, and MT-1MMP. Of these, MMP-2 and MT-1-MMP are the most studied for their role in angiogenesis. Very little is known about the specific in vivo substrates for the MMPs. However, from in vitro analysis of protease activity, it is evident that collectively the MMP family can degrade all known extracellular matrix components. These proteases are secreted as zymogens that must be activated in the extracellular compartment. The exception is MT-1-MMP, which is cell surface-bound and is processed prior to cellsurface localization by a furin-dependent mechanism (4, 7). Endogenous inhibitors known as the tissue inhibitors of metalloproteinases downregulate the activity of the MMPs. Currently, this family consists of four members, TIMP-1, TIMP-2, TIMP-3, and TIMP-4. The TIMPs are antiangiogenic but appear to have multiple effects on the angiogenic process and inhibition of MMP activity $(1,2)$.

The molecular mechanisms underlying the cellular events of angiogenesis have been examined utilizing a number of in vitro models. These have been helpful in examining the role of proteases in angiogenesis. They include growth of endothelial cells or vascular explants 
in amnionic membranes, fibrin clots, type I collagen, or basement membrane matrices. In these assays, endothelial cells acquire a migratory or invasive phenotype, reorganize the extracellular matrix, and in some cases recapitulate the tubular morphology of microvessels complete with lumen formation. In many early studies, the extracellular matrix was viewed as a barrier to endothelial cell invasion. The principal role of MMP activity was to remove this barrier and allow endothelial cell migration. Recent studies challenge this notion and suggest that cell-extracellular matrix interactions profoundly influence cell behavior. These interactions not only influence MMP production but are subject to modulation and regulation by MMP activity. In this way, MMP activity can directly and indirectly mediate the angiogenic response.

Treatment of endothelial cells with exogenous proMMP-2 induces a dose-dependent morphologic change consistent with an angiogenic response (i.e., tube formation) (8) However, this effect reaches a plateau, and addition of further MMP-2 begins to reverse tube formation. These effects are dependent on MMP-2 activity and are inhibited by TIMP-2. This suggests that exogenous pro-MMP-2 is activated by the endothelial cells. But how do endothelial cells activate pro-MMP-2?

Capillary endothelial cells cultured on twodimensional type I collagen gels produce low, constitutive levels of pro-MMP-2 with little endogenous activation of this protease. However, when placed in threedimensional type I collagen gels, there is a marked increase in pro-MMP-2 steady-state transcript levels (9). After three days in culture, the increase in MMP-2 secretion and activation is pronounced. This effect on MMP2 expression and activation is coordinate with enhancement of MT-1-MMP transcription and expression. The current model for MT-1-MMP-mediated activation of pro-MMP-2 involves TIMP-2 as a receptor (Figure 1a and ref. 7). Binding of pro-MMP-2 to the MT-1-MMP/TIMP2 complex localizes this pro-MMP- 2 on the cell surface, and activation is initiated by the proteolytic action of a second, TIMP-2-free MT-1-MMP molecule at the Asn ${ }^{37}$ $\mathrm{Leu}^{38}$ bond of the MMP-2 propeptide (10). Binding of pro-MMP-2 to TIMP-2 is mediated by the $\mathrm{COOH}$ terminal hemopexin-like domain (often referred to as PEX), present in most soluble MMPs. The structure of the PEX domain of MMPs consists of a four-bladed propeller composed of antiparallel $\beta$ sheets. The TIMP-2 binding domain is localized by mutational analysis to the junction of modules III and IV of the MMP-2 PEX domain (11). The study by Haas et al. (9) presents correlative data that supports a direct role for MT-1-MMP in the activation of pro-MMP-2 produced by endothelial cells cultured in three-dimensional collagen I gel. Culture of these cells on or in reconstituted basement membrane did not enhance MMP-2 production or MT-1MMP-mediated activation. This is an interesting contrast, as many view basement membrane as the first barrier that endothelial cells must cross to initiate an angiogenic response. Are other mechanisms of MMP activation operative in endothelial cells in contact with basement membrane and/or provisional matrix?

When rat endothelial cells are cultured for longer periods (five days) in three-dimensional type I collagen gels, there is evidence of endothelial cell organization into multicellular structures exhibiting lumen formation (9). Microvessel explants in three-dimensional cultures on type I collagen show evidence of endothelial sprout formation. The organization of endothelial cells into networks and sprout formation in microvessel explants are both inhibited by the inclusion of synthetic MMP inhibitors. It seems apparent that activation of MMP-2 synthesis in this model is mediated by integrin receptors, specifically $\alpha 2 \beta 1$. In other systems, $\alpha v \beta 3$ has also been implicated in initiating MMP-2 synthesis. However, as Haas et al. point out, the induction of MMP-2 in the rat endothelial cell model is not solely a response to a specific ligand (type I collagen) (9). The three-dimensional organization of the matrix and the response to mechanical forces in such a matrix are also important components of this response. Changes in cell shape (i.e., mechanical stress) are known to alter transcription of MMPs and other gene products; in fibroblasts, this is mediated by an autocrine mechanism (12). This raises issues regarding what specific integrin receptors initiate enhanced MMP production on different extracellular matrix substrates, what specific MMP profiles are induced, and how these signals are generated upon initiation of the angiogenic response.

Studies described above have focused on the role of MT-1-MMP as an MMP-2 activator in endothelial cell responses to angiogenic stimulation. However, recent findings suggest that, like the serine proteases, the role of specific MMPs may be contextual. Weiss and colleagues (4) used a combined in vitro/in vivo model system to study endothelial cell invasion of fibrin barriers. These investigators found that neoangiogenesis in a fibrin matrix was completely independent of uPA, tPA, or plasminogen, but was blocked by MMP inhibitors TIMP-2 or BB-94. Characterization of the endothelial cell MMP profile under conditions of angiogenic stimulation used in these studies revealed that only MT-1MMP possessed significant fibrinolytic activity. Transfection and expression of MT-1-MMP in a null cell line (MDCK cells) conferred fibrin-invasive potential to these cells. These findings suggest that the specific role MMPs may play in endothelial cell invasion may rely on the extracellular matrix that composes their environment.

The endothelial sprout might be expected to encounter a variety of extracellular matrices during vessel formation. Certainly, early events during the angiogenic response include detachment of endothelial cells from their underlying basement membrane and interaction with the provisional matrix formed by leakage of fibrinogen from the vascular compartment. After traversing these matrices, one can envision the tip of the endothelial sprout contacting interstitial extracellular matrix composed principally of type I collagen and fibronectin (Figure 2). The studies outlined above suggest that MT-1-MMP activation of pro-MMP-2 is critical for invasion of type I collagen matrix and that MT-1MMP alone is essential for traversing the provisional matrix surrounding leaky vessels $(4,9)$. However, it is not known if other MMPs or protease systems may also contribute to, or compensate for, MT-1-MMP and MMP-2. Consider that MT-1-MMP is only one of four currently known MMPs with a transmembrane domain. We know 


\section{Figure 1}

(a) MT-1-MMP activation of pro-MMP-2. As described in the text, TIMP-2 binding to the active site of MT-1-MMP and the PEX domain of pro-MMP-2 results in formation of a ternary complex. If this occurs in proximity to a second MT-1-MMP molecule, proteolytic modification of the pro-fragment of MMP-2 initiates activation of this protease. The activated protease may then dissociate from the cell surface and contribute to degradation of the extracellular matrix prerequisite for endothelial sprout invasion. In this mechanism TIMP-2 levels are critical for determining the level of pro-MMP-2 activation. TIMP-2 levels must be significantly lower than the local MT-1-MMP concentration to allow activation of MMP-2. TIMP-2 saturation of MT-1-MMP binding sites inhibits pro-MMP-2 activation via this MT-1-MMP mechanism. It is not known how free MT-1-MMP can recognize the pro-MMP-2-TIMP-2MT-1-MMP ternary complex and initiate activation. It is not known if MT-1MMP forms a noncovalent homo-dimer, or other complex, that may facilitate pro-MMP-2 activation. (b) Binding of MMP-2 to $\alpha v \beta 3$ integrin receptor. As described in the text, MMP-2 forms a stable complex with $\alpha v \beta 3$. This binding is mediated by the C-terminal PEX domain of MMP-2. Binding of recombinant PEX domain competes for binding of MMP-2 and inhibits angiogenesis. The mechanism of this inhibition is not known, but these findings suggest that MMP-2 activity may be required for endothelial cell detachment from stable matrix interactions. Moreover, the role of TIMP-2 in modulating the interaction of MMP- 2 with $\alpha v \beta 3$ is not known. It is possible that formation of the MMP-2-TIMP-2, which is mediated by interaction of TIMP-2 with the MMP PEX domain, may compete for binding of the protease to $\alpha v \beta 3$. Finally, the effects of MMP- 2 binding to $\alpha v \beta 3$ on signal transduction from this receptor are not known, nor are downstream consequences of possible changes in signaling events. (c) Interaction of MT-1-MMP and $\alpha v \beta 3$ with MMP-2 and TIMP-2 on the cell surface. As described in $\mathbf{a}$ and $\mathbf{b}$, there are at least two independent mechanisms for localization of MMP-2 on endothelial cell surface. Possible interactions between these two mechanism are illustrated as discussed in the text.

little about the possible contributions of MT-2-MMP, MT-3-MMP, or MT-4-MMP to angiogenesis.

An exciting finding regarding the role of MMPs in angiogenesis is the association of MMP-2 with the vitronectin receptor $\alpha v \beta 3$ integrin. For the first time, a direct link between protease activity and cell-matrix adhesive interactions is established. Brooks and colleagues $(13,14)$ are the first to report colocalization of MMP- 2 and $\alpha v \beta 3$ in vessels undergoing active remodeling in response to an angiogenic stimulus. These authors report a direct interaction of MMP- 2 with $\alpha v \beta 3$ involving the PEX domain of MMP-2 (Figure 1b). However, the mechanism of PEX binding to $\alpha v \beta 3$ remains unclear because MMP-2 lacks a RGD sequence that is common to many ligands for this receptor. It is also unclear if PEX is the only domain of MMP-2 that is involved in the binding, since a COOH-terminal fragment that extends in the $\mathrm{NH}_{2}$-terminal direction to include the flexible hinge region and third type II fibronectin repeat of MMP-2 (referred to as FHEX) binds poorly. This suggests that disruption of the tertiary structure of MMP-2 in the hinge region or fibronectin domain may interfere with binding to $\alpha v \beta 3$. Alternatively, the lack of structure at the $\mathrm{NH}_{2}$-terminal region of the larger FHEX may result in this hinge region being folded back into the PEX domain, disrupting binding.

The PEX domain lacks MMP-2 activity and competes for MMP-2 binding to $\alpha v \beta 3$ (13). PEX inhibits cell-surface type IV collagenolysis and angiogenesis in the chorioallantoic membrane assay. PEX inhibition of these activities correlates with a reduction of MMP-2 activation in vivo. Only active MMP-2 appears to be localized on the surface of
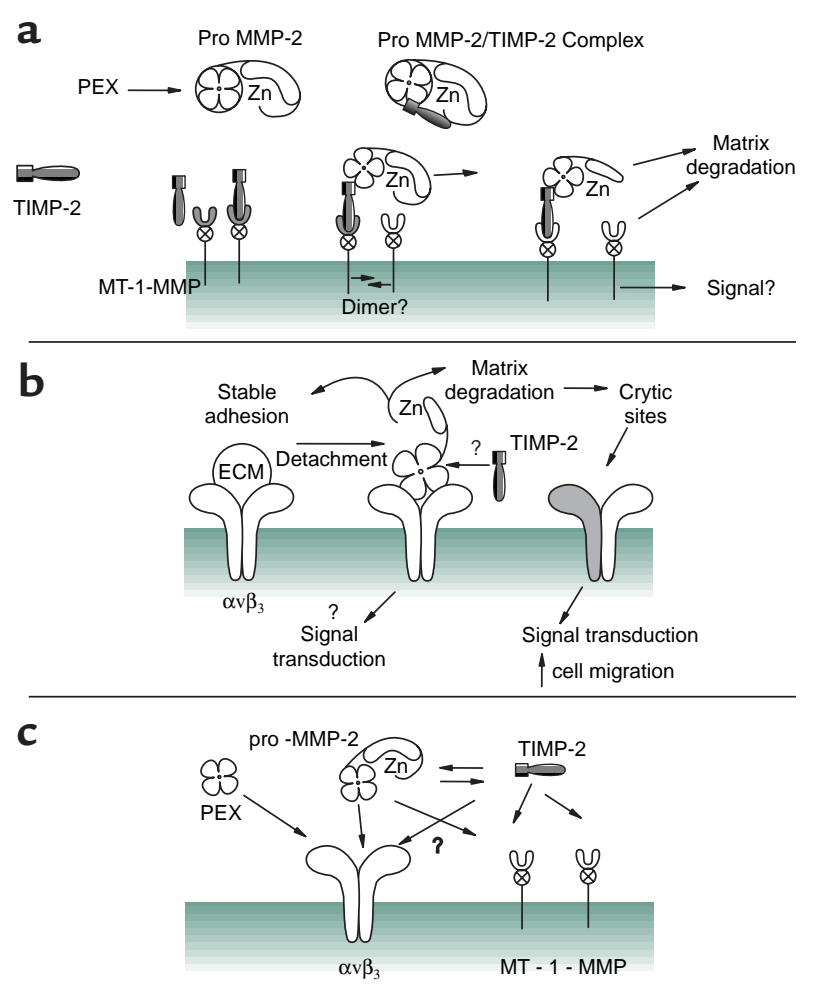

endothelial cells by $\alpha v \beta 3$ (14). In contrast to the MT-1MMP-dependent binding and activation of pro-MMP-2, interaction of MMP- 2 with $\alpha v \beta 3$ is independent of TIMP2 (Figure 1c). The question remains, how does the MMP-2 that binds to $\alpha v \beta 3$ become activated? The finding that PEX decreased MMP-2 activation is consistent with at least two possibilities. One possible scenario is $\alpha v \beta 3$ activates MMP-2 upon binding. The binding of TIMP-2 to the PEX domain of pro-MMP-2 may serve as a model for the activation by $\alpha v \beta 3$. Recent studies of TIMP- 2 binding to proMMP-2 observe an interaction between the inhibitor and the prodomain of MMP-2 $(11,15)$. This suggests that in the three-dimensional structure of the pro-MMP-2, the PEX domain may be in proximity with the prodomain. It is possible that upon binding of pro-MMP-2 to $\alpha v \beta 3$, there is a disruption in the prodomain that allows autoproteolytic activation of the enzyme to occur. In this model, PEX competes directly for pro-MMP-2 interaction with $\alpha_{v} \beta 3$ and thus prevents activation. An alternative mechanism entails MT-1-MMP-mediated activation of pro-MMP-2 and sub-sequent binding of the activated, TIMP-2-free MMP-2 to $\alpha v \beta 3$. The use of the PEX mutants that were utilized to define the TIMP-2 binding site on PEX should be useful to determine if the same sites are involved in MMP2 binding to $\alpha v \beta 3$. Alternatively, the influence of TIMP-2 on MMP- 2 binding to $\alpha v \beta 3$ should determine if similar sites on the PEX domain that bind TIMP-2 are involved in binding to $\alpha v \beta 3$. Does TIMP- 2 interaction with the PEX domain of MMP- 2 compete for binding to the $\alpha v \beta 3$ receptor? Is $\alpha v \beta 3$-bound MMP-2 inhibited by TIMP-2? Does $\alpha v \beta 3$-bound MMP-2 interact with MT-1-MMP?

It is interesting to note that multivalent ligation of the $\alpha_{v} \beta 3$ receptor in melanoma cells is known to result in transcriptional activation of MMP-2 and secretion of MMP-2 as well as TIMP-2 (16). In these reports, 


\section{Figure 2}

Model of tumor-induced changes in extracellular matrix during angiogenesis. Tumor-associated angiogenic factors induced enhanced vascular permeability, resulting in disruption of the subendothelial basement membrane and formation of a fibrin-rich provisional matrix. The endothelial cell responding to this stimulus must traverse not only the subendothelial basement membrane but also this provisional matrix. Moreover, the type I collagen and fibronectin containing interstitial matrix forms a third distinct type of extracellular matrix that endothelial cells recruited to support tumor growth must cross. During the angiogenic response endothelial cells must penetrate several types of extracellular matrix. MMP activity is required for this response. As discussed in the text, the role of specific MMPs, such as MT-1-MMP, may change as endothelial cells come in contact with different extracellular matrix environments. Moreover, the type of extracellular matrix may alter the profile of MMP expression. Studies on these effects may identify extracellular matrix-specific roles for different members of the MMP family. Such information would be useful for selecting the appropriate MMP for targeting with selective synthetic MMP inhibitors.

enhanced MMP-2 production correlates with increased in vitro melanoma cell invasiveness. This response is not observed using RGD peptides. It is not known if endothelial cells respond similarly by increasing MMP-2 production upon ligation of the $\alpha v \beta 3$ receptor. More specifically, it would be of interest to know if MMP-2 binding to $\alpha_{v} \beta 3$ can induce endothelial cell MMP-2 expression. This would create a positive-feedback loop in which localization of MMP- 2 on the cell surface by $\alpha v \beta 3$ mediated binding results in enhanced cellular production of MMP-2, and in turn, localization of more MMP2 on the cell surface. Once a threshold level of MMP-2 binding to the cell surface is reached, sufficient alterations in endothelial cell-matrix interactions or production of sufficient matrix degradation products may be achieved and initiate endothelial cell migration.

MMP-2 may facilitate endothelial cell invasion by removing matrix barriers or initiating signaling pathways that promote or support the angiogenic phenotype. Recent findings demonstrate that MMP-2 activity may generate extracellular matrix degradation fragments, leading to signals required for cell survival and/or migration. The selective action of MMP-2 on laminin-5 is known to result in stimulation of mammary epithelial cell migration (17). This finding suggests that similar MMP-generated degradation products of the extracellular matrix could influence endothelial cell behavior. The $\alpha v \beta 3$ integrin is known to mediate endothelial cell sur-

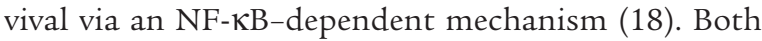
MMP-2 and TIMP-2 can modulate cell growth (1). Is MMP-2 binding to the $\alpha_{v} \beta 3$ receptor the mechanism that alters signal transduction and/or endothelial cell survival and proliferation? Does binding of MMP-2 via $\alpha v \beta 3$ alter endothelial cell attachment to the extracellular matrix? Does TIMP-2 suppress endothelial cell growth (1) by competing for MMP-2 binding to $\alpha v \beta 3$ ?

Endogenous PEX is found to accumulate at sites of $\alpha v \beta 3$ expression and vessel maturation (13). This suggests that endogenous PEX acts as a natural inhibitor of MMP-2 cell-surface localization and activation, thereby regulating the invasive behavior of new blood vessels. This mechanism of PEX downregulation of the angiogenic response may follow a number of different scenarios. One possible mechanism is that endothelial cells respond to the reduction of MMP-2-mediated matrix proteolysis by sensing a change in MMP-2 binding to $\alpha v \beta 3$, i.e., that PEX binding is not functionally equivalent to MMP-2 binding. This may be through a coreceptor or altered affinity for $\alpha v \beta 3$. The change in ligand affinity could alter signal transduction pathways supporting continued endothelial cell invasion. Alternatively, PEX binding may prevent the MMP-2 localization and generation of matrix degradation fragments that supports the angiogenic phenotype or preliminary organization of the matrix. In the absence of MMP-2 activity, the balance shifts in favor of angiogenesis inhibitors. This raises several questions. What is the mechanism for generation of endogenous PEX, and how is this process initiated or controlled? Is this the result of autoproteolytic degradation of active MMP-2, or is some other protease involved? Can the system be manipulated to increase endogenous PEX production and to shut down the pathologic angiogenic response? Interestingly, a recent report describes a $\mathrm{COOH}$-terminal fragment of MMP-11 (stromeysin-3) in culture medium from cocultures of non-small-cell lung cancer cells and normal pulmonary fibroblasts (19). Like PEX, this naturally occurring MMP fragment has no protease activity. A regulatory role in matrix turnover is suggested, but a specific biologic activity of this $35-\mathrm{kDa}$ COOH-terminal fragment of MMP-11 is not described.

Another possible role for MMPs in the angiogenic response is demonstrated in MMP-9-deficient mice (5). These mice exhibit abnormal growth plate vascularization and ossification. This study demonstrates that the abnormality in the growth plate vascularization in the MMP9-deficient mice is due to failure to release an unidentified angiogenic factor from the matrix, or alternatively, failure to effect degradation of an angiogenesis inhibitor that is present in the matrix. Similarly, MMP-1, MMP-3, as well as plasmin and heparanase, degrade endothelial-derived perlecan and release bound basic fibroblast growth factor (20). This potential mechanism of MMP-mediated growth factor release during tumor-induced angiogenesis has long been postulated but remains to be demonstrated.

Interestingly, MMP activity is now thought to be the principal protease activity responsible for generating the potent angiogenesis inhibitor angiostatin $(21,22)$. MMPs can degrade plasminogen to generate an $\mathrm{NH}_{2}$-terminal fragment that inhibits endothelial cell proliferation and is known as angiostatin. These include MMP-3, 
MMP-7, MMP-9, and MMP-12. MMP-12 is the most potent in generating angiostatin, which inhibits microvascular endothelial cell proliferation in vitro (21, 23). Angiostatin production also correlates with MMP12 synthesis in macrophages cultured from Lewis lung tumors grown in mice (23). Another recently identified inhibitor of angiogenesis is endostatin, which is a proteolytic fragment of collagen XVIII (24). It is unknown if MMP activity contributes to the generation of endostatin activity. What is the role of MMPs in generating angiogenesis inhibitors during endothelial sprout formation? Clearly, differentiation and suppression of endothelial cell proliferation occurs in the proximal region of the endothelial sprout as elongation proceeds. Does MMP activity, known to be active in generating angiogenesis inhibitors such as angiostatin, mediate this shift in endothelial cell phenotype? Are MMPs involved in generating the proteolytic fragments that act as inhibitors of angiogenesis?

MMPs are involved in more than the breakdown of connective tissue barriers necessary for new vessel formation. They also function to promote angiogenesis by regulating endothelial cell attachment, proliferation, migration, and growth, either directly or by release of growth factors sequestered in the extracellular matrix. Moreover, new evidence suggests that MMPs may also generate or release angiogenesis inhibitors such as angiostatin from the extracellular matrix. MT-1-MMP and MMP-2 are involved immediately at the endothelial cell surface and function immediately in extracellular matrix turnover and regulation of cell-extracellular matrix interactions. These changes in turnover of the extracellular matrix may result in phenotypic changes associated with the angiogenic response. Cells other than endothelial cells (i.e., tumor macrophages) secrete MMP-9 or MMP-12. The action of these proteases may indirectly influence endothelial cell behavior by release of proangiogenic factors, destruction of angiogenesis inhibitors, or generation of matrix fragments that inhibit angiogenesis, i.e., angiostatin or endostatin.

MMP activity is an early event in the angiogenic response, and recent findings suggest that this activity may directly influence endothelial cell behavior. New evidence suggests that MMPs may facilitate angiogenesis as well as function to generate angiogenesis inhibitors. Significant effort is directed at identifying the MMPs that mediate the angiogenic response for the purpose of therapeutic targeting of their activity to disrupt tumor neovascularization and subsequent dissemination (metastasis formation). In light of recent evidence demonstrating the dual role for MMPs in angiogenesis, it is imperative that we correctly identify and selectively target the appropriate MMPs. Can we make highly selective synthetic MMP inhibitors that eliminate potential side effects? Or does disruption of noncatalytic activities (i.e., PEX inhibitors) present a safer strategy for targeting angiogenesis? Does upregulation of specific MMP activity to generate endogenous angiogenesis inhibitors present a reasonable alternative? Clearly, MMPs are integral to the process of angiogenesis, but much work is needed to score the most effective therapeutic hit for these moving targets.

\section{Acknowledgments}

I am grateful to Lance A. Liotta and Maryalice Stetler-Stevenson for their critical reading of the manuscript and helpful discussions.

1. Murphy, A.N., Unsworth, E.J., and Stetler-Stevenson, W.G. 1993. Tissue inhibitor of metalloproteinase-2 inhibits bFGF-induced human microvascular endothelial cell proliferation. J. Cell. Physiol. 157:351-358.

2. Anand-Apte, B., et al. 1997. Inhibition of angiogenesis by tissue inhibitor of metalloproteinase-3. Invest. Ophthalmol. Vis. Sci. 38:817-823.

3. Benelli, R., et al. 1994. Inhibition of AIDS-Kaposi's sarcoma cell induced endothelial cell invasion by TIMP-2 and a synthetic peptide from the metalloproteinase propeptide: implications for an anti-angiogenic therapy. Oncol. Res. 6:251-257.

4. Hiraoka, N., Allen, E., Apel, I.J., Gyetko, M.R., and Weiss, S.J. 1998. Matrix metalloproteinases regulate neovascularization by acting as pericellular fibrinolysins. Cell. 95:365-377.

5. Vu, T.H., et al. 1998. MMP-9/gelatinase B is a key regulator of growth plate angiogenesis and apoptosis of hypertrophic chondrocytes. Cell. 93:411-422.

6. Itoh, T., et al. 1998. Reduced angiogenesis and tumor progression in gelatinase A-deficient mice. Cancer Res. 58:1048-1051.

7. Werb, Z. 1997. ECM and cell surface proteolysis: regulating cellular ecology. Cell. 91:439-442.

8. Schnaper, H.W., et al. 1993. Type IV collagenase(s) and TIMPs modulate endothelial cell morphogenesis in vitro. J. Cell. Physiol. 156:235-246.

9. Haas, T.L., Davis, S.J., and Madri, J.A. 1998. Three-dimensional type I collagen lattices induce coordinate expression of matrix metalloproteinases MT1-MMP and MMP-2 in microvascular endothelial cells. J. Biol. Chem. 273:3604-3610.

10. Will, H., Atkinson, S.J., Butler, G.S., Smith, B., and Murphy, G. 1996. The soluble catalytic domain of membrane type 1 matrix metalloproteinase cleaves the propeptide of progelatinase A and initiates autoproteolytic activation. Regulation by TIMP-2 and TIMP-3. J. Biol. Chem. 271:17119-17123.

11. Overall, C.M., et al. 1999. Identification of the tissue inhibitor of metalloproteinases-2 (TIMP-2) binding site on the hemopexin carboxyl domain of human gelatinase a by site-directed mutagenesis. The hierarchical role in binding TIMP-2 of the unique cationic clusters of hemopexin modules III and IV. J. Biol. Chem. 274:4421-4429.

12. Kheradmand, F., Werner, E., Tremble, P., Symons, M., and Werb, Z. 1998. Role of Rac1 and oxygen radicals in collagenase- 1 expression induced by cell shape change. Science. 280:898-902.

13. Brooks, P.C., Silletti, S., von Schalscha, T.L., Friedlander, M., and Cheresh, D.A. 1998. Disruption of angiogenesis by PEX, a noncatalytic metalloproteinase fragment with integrin binding activity. Cell. 92:391-400.

14. Brooks, P.C., et al. 1996. Localization of matrix metalloproteinase MMP-2 to the surface of invasive cells by interaction with integrin $\alpha v \beta 3$. Cell. 85:683-693.

15. Olson, M.W., Gervasi, D.C., Mobashery, S., and Fridman, R. 1997. Kinetic analysis of the binding of human matrix metalloproteinase- 2 and -9 to tissue inhibitor of metalloproteinase (TIMP)-1 and TIMP-2. J. Biol. Chem. 272:29975-29983.

16. Bafetti, L.M., Young, T.N., Itoh, Y., and Stack, M.S. 1998. Intact vitronectin induces matrix metalloproteinase- 2 and tissue inhibitor of metalloproteinases-2 expression and enhanced cellular invasion by melanoma cells. J. Biol. Chem. 273:143-149.

17. Giannelli, G., Falk-Marzillier, J., Schiraldi, O., Stetler-Stevenson, W.G., and Quaranta, V. 1997. Induction of cell migration by matrix metalloprotease-2 cleavage of laminin-5. Science. 277:225-228.

18. Scatena, M., et al. 1998. NFKB mediates $\alpha v \beta 3$ integrin-induced endothelial cell survival. J. Cell Biol. 141:1083-1093.

19. Mari, B.P., et al. 1998. Stromelysin-3 is induced in tumor/stroma cocultures and inactivated via a tumor-specific and basic fibroblast growth factor-dependent mechanism. J. Biol. Chem. 273:618-626.

20. Whitelock, J.M., Murdoch, A.D., Iozzo, R.V., and Underwood, P.A. 1996. The degradation of human endothelial cell-derived perlecan and release of bound basic fibroblast growth factor by stromelysin, collagenase, plasmin, and heparanases. J. Biol. Chem. 271:10079-10086.

21. Cornelius, L.A., et al. 1998. Matrix metalloproteinases generate angiostatin: effects on neovascularization. J. Immunol. 161:6845-6852.

22. Patterson, B.C., and Sang, Q.A. 1997. Angiostatin-converting enzyme activities of human matrilysin (MMP-7) and gelatinase B/type IV collagenase (MMP-9). J. Biol. Chem. 272:28823-28825.

23. Dong, Z.Y., Kumar, R., Yang, X.L., and Fidler, I.J. 1997. Macrophagederived metalloelastase is responsible for the generation of angiostatin in Lewis lung carcinoma. Cell. 88:801-810.

24. O'Reilly, M.S., et al. 1997. Endostatin: an endogenous inhibitor of angiogenesis and tumor growth. Cell. 88:277-285. 